\title{
Automatic Identification of a Coughing Animal using Audio and Video Data
}

\author{
Heegon Kim ${ }^{1 a}$, Jaewon Sa ${ }^{b}$, Byeongjoon Noh ${ }^{\mathrm{c}}$, Jonguk Lee \\ Dept. of Computer and Information Science, Korea University, Sejong, 339-700, Korea \\ E-mail: ${ }^{a}$ khg86@korea.ac.kr; ${ }^{b}$ sjwon92@korea.ac.kr; ${ }^{c}$ powernoh@korea.ac.kr; \\ deastwest9akorea.ac.kr
}

\section{Yongwha Chung ${ }^{2}$}

Dept. of Computer and Information Science, Korea University, Sejong, 339-700, Korea

E-mail: ychungy@korea.ac.kr

\section{Daihee Park ${ }^{3}$}

Dept. of Computer and Information Science, Korea University, Sejong, 339-700, Korea

E-mail: dhpark@korea.ac.kr

\begin{abstract}
Automatic detection of individual animal's health condition based on a 24-hour monitoring system is important for efficient management of a livestock farm. In particular, the wasting disease is highly contagious in the group-based environment. The early detection of wasting disease is very important in order to minimize possible damage to the farm. In this paper, we propose a method to detect the wasting disease automatically by using both audio and video data. When a cough sound is detected with audio analysis, the Motion History Image-based video analysis will identify the coughing animal. By computing the change of MHI motion and the moving distance of each moving animal, the coughing animal can be identified. Based on the experimental results, we confirm that the Motion History Image-based method can discriminate the shaking motion of a coughing from other movement motions with careful motion pattern analysis.
\end{abstract}

ISCC 2015

18-19, December, 2015

Guangzhou, China

\footnotetext{
${ }^{1}$ Speaker

${ }^{2}$ Corresponding Author

${ }^{3}$ This research was supported by Basic Science Research Program through the NRF funded by the MEST(2015R1D1A1A09060594) and BK21 Plus Program.
} 


\section{Introduction}

Early detection of the animal's wasting disease is important for caring the group-housed animals because the respiratory diseases are one of the main causes of mortality and loss of productivity in the group-housed animal farming [1]. When the wasting diseases are undetected or are detected late, the profitability of the farms can be significantly affected.

Coughing is a central element to diagnose common respiratory disease. Clinically, coughing is the most frequently presented symptom of many diseases that may affect the airways and lungs, and it is often an early symptom of respiratory disease [2]. It is one of the body's defense mechanisms against respiratory infections and it can be a sign of a disorder of or infection in the respiratory system. This sound is so characteristic that it is possible to distinguish a cough from other vocal manifestations [1]. Also, the audio analysis is of considerable importance because the audio production by animals is a candidate bio-signal that can be easily measured "at a distance" without causing any additional stress to the animals [34]. Furthermore, in recent years, the audio analysis has become an increasingly important tool to interpret behavior, health conditions and well-being of animals [5-6].

Various studies are researched by using audio data obtained from animal's room [1][6][7] [8]. Although the current solutions can detect coughing in a group-housed room, a coughing animal in the room cannot be identified with the current solutions. Automatic identification of the coughing animal is very important in order to minimize the possible damage to the grouphoused farm. In this paper, we propose a method to identify a coughing animal automatically by using both audio and video data. When a cough sound is detected with audio analysis, the Motion History Image(MHI)-based video analysis will identify the coughing animal[9]. On the basis of careful analysis of the motion pattern, the MHI-based method can discriminate the shaking motion of a coughing from other movement motions. Note that, we may use some "attached" sensors in order to identify a coughing animal; however, these attached sensors can cause false alarms when the sensors are bumped into a structure or other animals in a room. Moreover, these sensors are relatively expensive for large-scale farms. We must reduce the managing cost in large-scale farms that employ individual sensors and eliminate the requirement of farm workers having to verify individual sensors. Based on the experimental results, we confirm that a coughing animal can be identified with both "non-attached" audio and video sensors successfully.

\section{Related Works}

\subsection{Cough Detection by Using Audio Data}

For animal's disease detection using audio data, various studies have been reported. One study classified several pig wasting diseases with features such as pitch, intensity, duration, and formants 1, 2, 3 and 4 of the collected cough samples [1]. Another study discriminated cough audio from other audios (e.g., metal rack audios), and indicated automatic on-line monitoring tool for cough recognition [10]. Other studies could classify the coughing audios of pigs by using Hidden Markov Models [11] and artificial intelligence [12]. These studies are useful in early detection of possible disease. Although these studies can detect coughing by using audio 
data, they cannot identify a coughing animal. In order to reduce the possible damage, we need to identify the coughing animal accurately.

\subsection{MHI by Using Video Data}

For animal's behavior detection by using video data, a frame difference method can be adopted. The frame difference method is a simple method, which compares the number of pixels between previous frame and current frame. When difference of pixel is detected by using the frame difference method, the detected area can be decided which animal has a movement; however, this method cannot identify the coughing animal.

MHI is an efficient technique for action representation. MHI is presented as a relatively new concept where the moving parts of a video sequence can be engraved in a single image allowing the prediction of motion flow and the moving portions of the video action. This action/motion representation technique has become popular owing to its simple algorithm and ease of implementation. Consequently, MHI representations for numerous applications and MHI technique for action recognition have become fashionable in the computer vision community [13].

MHI can describe motion in a sequence of continuous frames. The silhouette sequence is condensed into a gray scale image; the most recent motion in a frame is encoded as the brightest gray value; older motion is represented as a darker gray value (compared to the gray value of the latest motion). MHI represents a gray value as black when there is no motion in the frame [9]. Fig. 1 shows the result of applying MHI to a pig monitoring application. Since MHI can represent the shaking motion of a coughing, we apply MHI to identify a coughing pig.

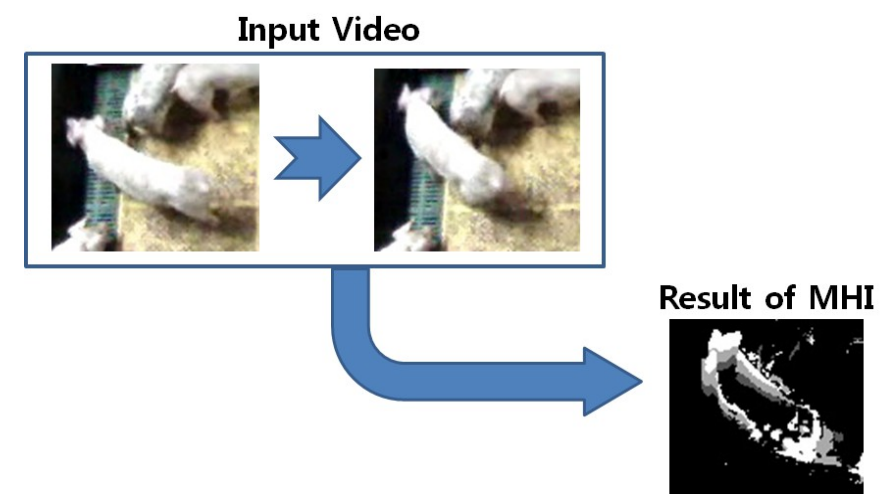

Figure 1: Result of Motion History Image

\section{Automatic Identification of A Coughing Animal}

\subsection{Proposed System}

In this paper, we propose a new system for automatic identification of coughing animals using both audio and video data, and we can identify which coughing animals are in a room. Algorithm 1 shows the proposed algorithm. Fig. 2 represents an overview of the proposed identification system. When a cough sound is detected, MHI-based video analysis identifies the coughing animal. 
Algorithm 1. Identification of A Coughing Animal
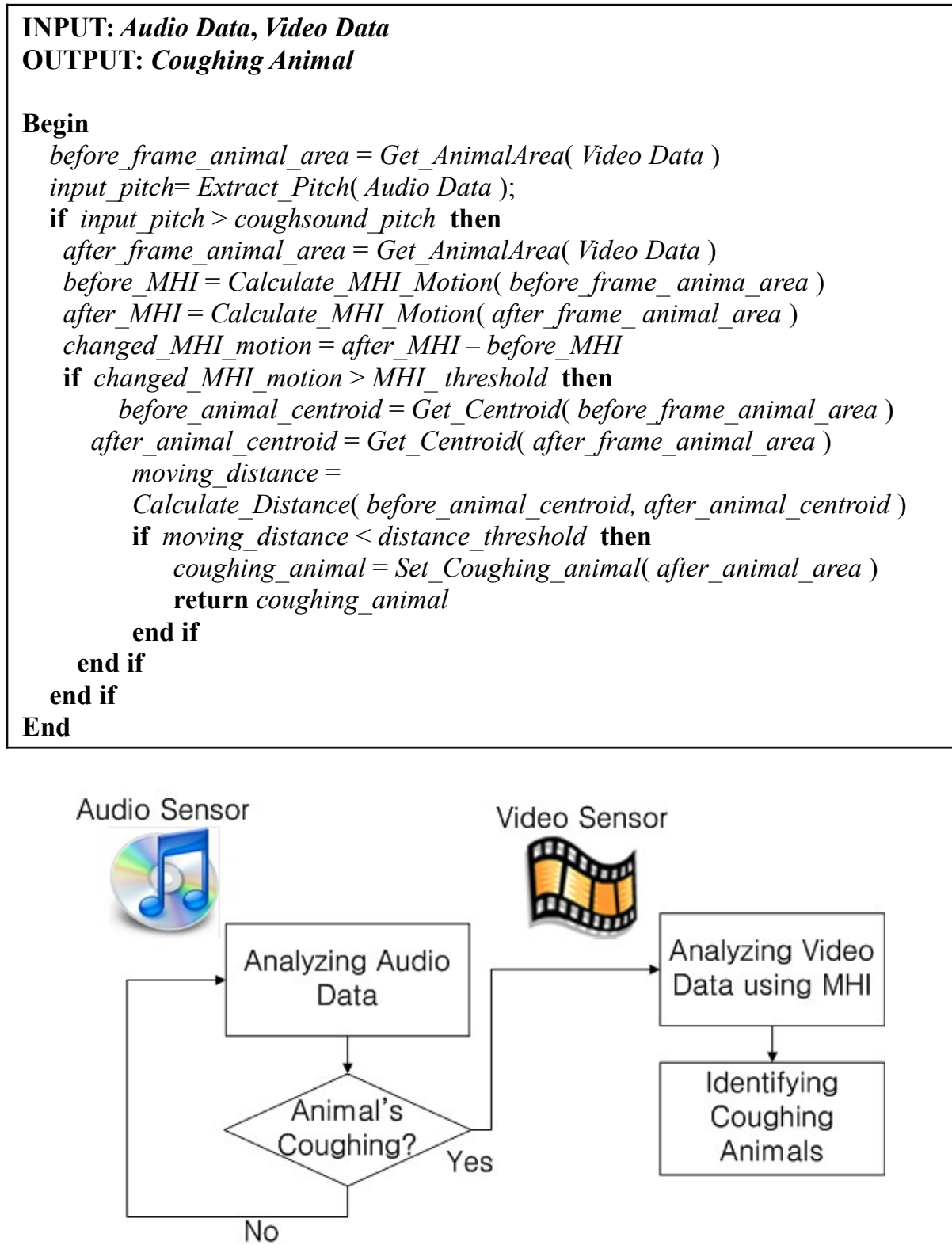

Figure2: Overview of the Automatic Identification System of A Coughing Animal.

\subsection{Detection of Animal's Coughing using Audio Data}

For efficient coughing animal identification, a cough sound detection is executed prior to the video analysis (i.e., video analysis is executed only if a cough sound is detected). The cough sound detection uses average pitch which is one of the sound features, and pitch is extracted from collected audio data per second. If the pitch value is within range of a cough sound threshold, our proposed system decides that coughing animals are in the room [7]. 


\subsection{Identification of A Coughing Animal by Using Video Data}

For identifying a coughing animal, the video data is analyzed by using MHI. When the video is analyzed by using the frame difference method, a coughing animal cannot be discriminated a fast moving animal because both animals have lots of motion. On the other hand, if the video is analyzed by using MHI, the coughing animals can be identified by analyzing the shaking motion. The coughing animal stops the movement during coughing, whereas the fast moving animal has constant movement. Thus, a candidate of the coughing animal can be identified by using the amount of a momentary motion, and the amount of the momentary motion (denoted as amount of MHI motion) is calculated by using the difference of MHI-based motion between a previous frame and a current frame.

After identifying a candidate of the coughing animal, a centroid of the candidate is analyzed. The centroid difference of a coughing animal between successive frames (denoted as moving distance of centroid) is negligible because the coughing animal stops his movement at the shaking motion during coughing; therefore, a coughing animal has a large value of the amount of MHI motion and a small value of the moving distance of centroid.

\section{Experimental Results}

The experiment was conducted with audio and video data obtained from a commercial pig production farm located in Chungnam province, South Korea. Fig. 3 shows the pig room where the audio and video sensors were installed. The resolution of video was $640 \times 480$ and the time duration of MHI was set to three frames.

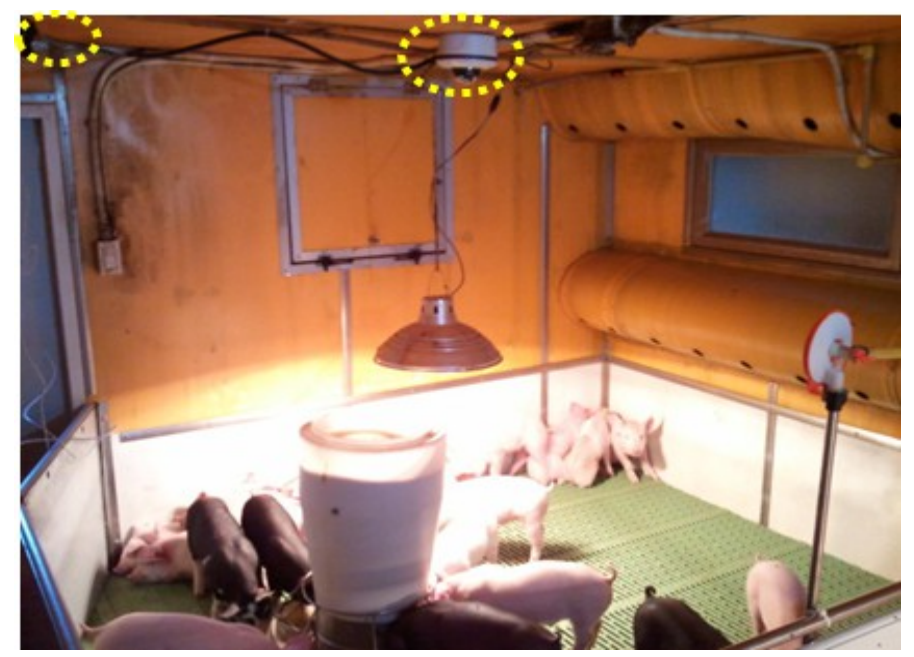

Figure 3: Installed Audio and Video Sensors in A Pig Room.

Fig. 4 shows the amount of MHI motion when a cough sound was detected at frame \#4. In video \#1 and video \#2, there were two moving pigs, in addition to a coughing pig. We denote a moving pig with larger (smaller) movement motion computed with MHI as a fast(slow) moving pig, as shown in Fig. 4. As the amount of MHI motion of a coughing pig was smaller than that of the fast moving pig and larger than that of the slow moving pig during frame \#4 \#6, a 
simple comparison of the amount of MHI motion could not discriminate the coughing pig from the moving pigs.

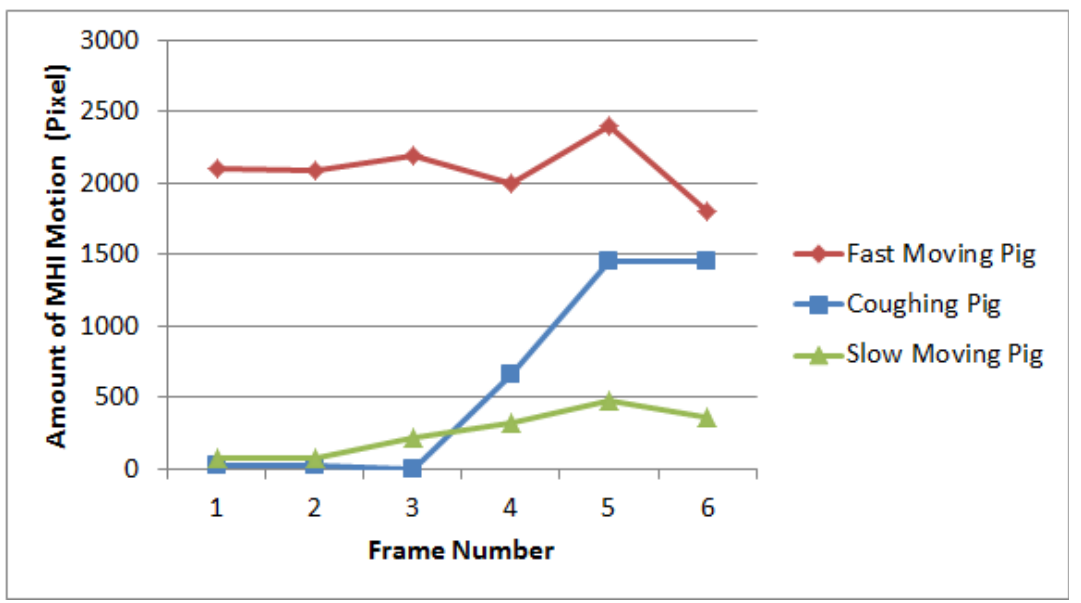

(a)video \#1

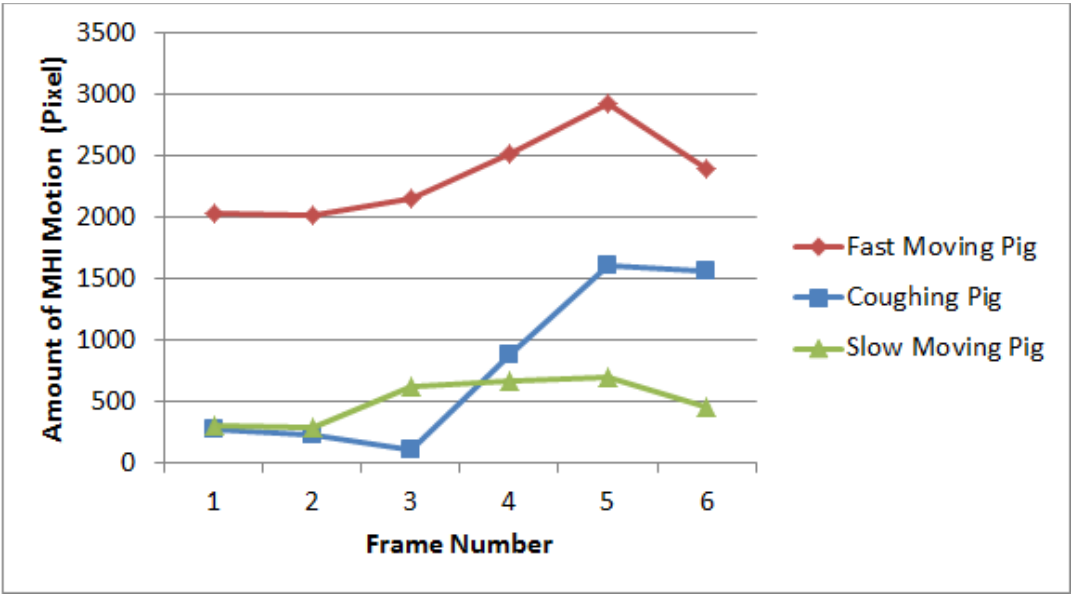

(b)video \#2

Figure 4: Amount of MHI Motion When A Cough Sound Was Detected.

For discriminating the coughing pig from the moving pigs, the change of MHI motion was calculated. The change of MHI motion of the coughing pig was larger than those of the moving pigs, as shown in Fig. 5; however, the change of MHI motion of the fast moving pig was relatively large in video $\# 2$, and thus we need to check the moving distance of centroid. 


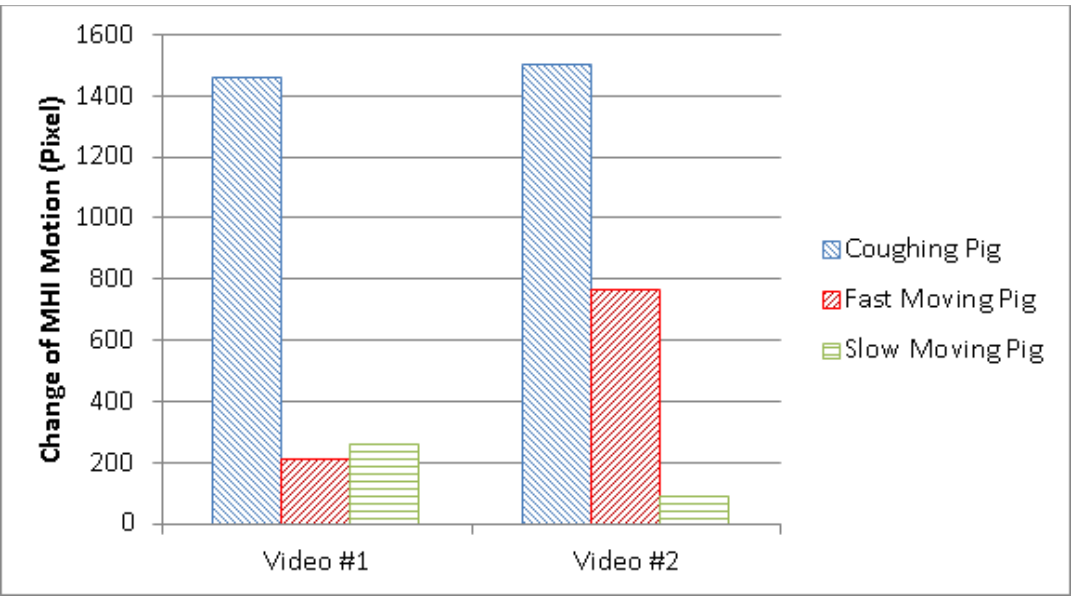

Figure 5: Change of MHI Motion.

As shown in Fig. 6, the moving distance of centroid of the fast moving pig in video \#2 was much larger than that of the coughing pig in video \#2. In fact, the shaking motion of a coughing pig should have the large change of MHI motion and the small moving distance of centroid. If we check the change of MHI motion (i.e., larger than 1000) and the moving distance of centroid (i.e., smaller than 5), we can identify a coughing pig.

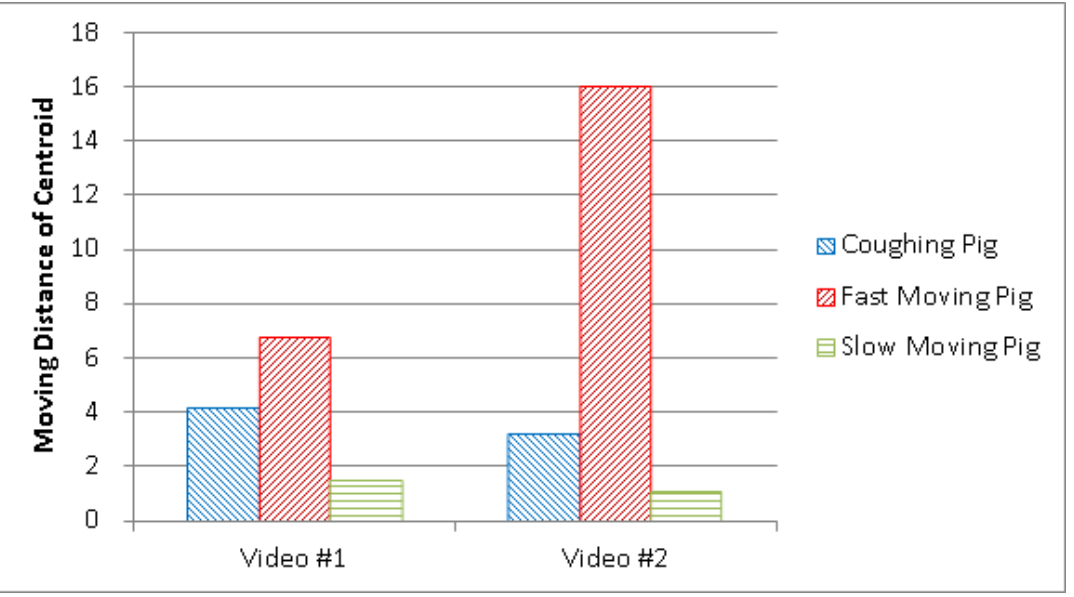

Figure 6: Moving Distance of Centroid.

\section{Conclusion}

The early detection of the animal's wasting disease is important because many animals in a group-housed farm can be damaged by the infectious disease. In this paper, we proposed a method to identify a coughing animal by analyzing both audio and video data. When a cough sound is detected with audio analysis, the MHI-based video analysis detects the shaking motion of a coughing. Based on the experimental results, we confirm that the proposed method can identify a coughing animal in a group-housed farm by using both audio and video data. 


\section{References}

[1]W. M. Gutierrez, S. Kim, D. Kim, S. Yeon, and H. Chang, Classification of porcine Wasting Diseases using Sound Analysis, AJAS. 23(8): 1096-1105(2010)

[2]J. Hirschberg and T. Szende, Pathological Cry, Stridor and Cough in Infants, Akiademiai Kiado. (1982)

[3]J. M. Aerts, P. Jans, D. halloy, P. Gustin, and D. Berckmans, Labelin of Cough Data from Pigs for On-line Disease Monitoring by Sound Analysis, Transactions-American Society of Agricultural Engineers. 48(1): 351-354(2005)

[4]D. J. Moura, I. A. Naas, E. C. S. Alves, T. M. R. Carvalho, M. M. Vale, and K. A. O. Lima, Noise Analysis to Evaluate Chick Thermal Comfort, Scientia Agricola. 65(4): 438-443(2008)

[5]Y. Chung, J. Lee, S. Oh, D. Park, H. Chang, and S. Kim, Automatic Detection of Cow's Oestrus in Audio Surveillance System, AJAS. 26(7): 1030-1037(2013)

[6]Y. Chung, S. Oh, J. Lee, D. Park, H. Chang, and S. Kim, Automatic Detection and Recognition of Pig Wasting Diseases using Sound Data in Audio Surveillance Systems, Sensors. 13(10): 1292912942(2013)

[7]J. Lee, L. Jin, D. Park, Y. Chung, and H. Chang, Acoustic Features for Pig Wasting Disease Detection, IJIPM. 6(1): 37-46(2015)

[8]V. Exadaktylos, M. Silva, and D. Berckmans, Real-time analysis of chicken embryo sounds to monitor different incubation stages, Computers and Electronics in Agriculture. 75(2): 321$326(2011)$

[9]A. Bobick and J. Davis, The Recognition of Human Movement using Temporal Templates, IEEE PAMI. 23(1): 257-267(2001)

[10]S. Ferrari, R. Piccinini, M. Silva, V. Exadaktylos, D. Berckmans, and M. Guarino, Cough sound description in relation to respiratory diseases in dairy calves, Preventive Veterinary Medicine. 96(3): 276-280(2010)

[11]A. L. Giesert, W. T. Balke, and G. Jahns, Probabilistic analysis of coughs in pigs to diagnose respiratory infections, Landbauforschung vTI Agriculture and Forestry Research. 3(61): 237 242(2011)

[12]N. Risi, K. O. Silva, P. R. F. Zulato, R. C. Guido, and G. Borges, Use of Artificial Intelligence to Identify Vocalizations Emitted by Sick and Healthy Piglets, Proc. of International Livestock Environment Symposium. 8(1): (2008)

[13]A. Ahad, Motion History Images for Action Recognition and Understanding, Springer Briefs in Computer Science. (2013) 